\title{
Receiver Design for Downlink MIMO MC-CDMA in Cognitive Radio Systems
}

\author{
M. Rajabzadeh ${ }^{1,2}$ and H. Khoshbin ${ }^{1}$ \\ ${ }^{1}$ Electrical Engineering Department, Ferdowsi University of Mashhad, Mashhad, Iran \\ ${ }^{2}$ Razavi House of Elites, Mashhad, Iran
}

\begin{abstract}
Multiuser interference (MUI) and co-space interference (CSI) are two challenging issues in design of multicarrier code-division multiple-access (MC-CDMA) systems when multiple antennas are used at both the transmitter and receiver sides (MIMO transmission). On the other hand, in cognitive radio (CR) system, the secondary users that use MCMCDA technique must deactivate the subcarriers used by the primary users. This leads to loss of orthogonality of the conventional spreading codes such as Hadamard Walsh codes. In this paper, we have modified two recently proposed linear receivers to be deployed in MIMO MC-CDMA based CR downlink transmission where some of the subcarriers are turned off. To solve the resultant non-orthogonality problem, the complex carrier interferometry $(\mathrm{CI})$ codes are utilized that exist for any length. The first method, called total interference cancellation (CI-TIC), is able to completely eliminate the CSI and the MUI. The other method is called CI symbol-chip level minimum mean square error (CI-SC-MMSE) that shows superior bit error performance with respect to CI-TIC. For different scenarios, simulation results show that the proposed CI based receivers are able to effectively mitigate the MUI and the CSI in comparison with the case where Hadamard Walsh codes are utilized.
\end{abstract}

Index Terms - cognitive radio, downlink MC-CDMA, MIMO, SVD, space-frequency spreading, MMSE.

\section{INTRODUCTION}

Cognitive radio is a novel approach for improving the utilization of the scarce radio spectrum by making it possible for a group of secondary users (SU) to access the frequency bands which are not used by the primary users (PU). As the unused spectrums may be non-contiguously available for the secondary users, multicarrier techniques such as OFDM and multicarrier code division multiple access (MC-CDMA) are good candidates to efficiently utilize these spectrum holes[1]. MC-CDMA system is able to mitigate the intersymbol interference (ISI) and the multiuser interference (MUI) in the frequency selective channels because of combining orthogonal frequency division multiplexing (OFDM) technique and orthogonal spreading codes.

Benefitting the transmission through multiple antennas, multiple-input multiple-output (MIMO) MC-CDMA systems have attracted many attentions for being deployed in broadband wireless communications for attaining more capacity[2]. However, the arising co-space interference (CSI) due to MIMO transmission ruins the orthogonality of the spreading codes that causes multiuser interference (MUI).

To address these challenging issues, a multiple of effective linear [2,3] and nonlinear [4] receivers have been proposed. The chip level ordered successive spatial and multiuser interference cancellation (OSSMIC) receiver [4] is a modified V-BLAST based receiver to reject the spatial and multiuser interference jointly. The linear total interference cancellation (TIC) receiver, proposed in [3], is based on the singular value decomposition (SVD) of the channel matix. The TIC completely eliminates the CSI and MUI by using orthogonal Walsh spreading codes, however, its performance suffers from the small singular values of the channel. So another linear SVD based receiver has been proposed that is named as symbol chip level MMSE (SC-MMSE) [3] which solves this problem by minimizing the MSE criterion. It also relys on orthogonal spreading codes.

To use MC-CDMA in cognitive radio transmission, the SU base station can only deploy the non-contiguous spectrum holes by turning off the subcarriers that are within the spectrum used by the PUs. Such system is called noncotiguous (NC) MC-CDMA [5,6]. In this case, the number of available subcarriers could be any integer values. The conventional orthogonal Hadamard-Walsh (HW) codes only exist when their length is multiple of four. So these codes cannot provide the desired orthogonality in $\mathrm{CR}$ environment.

In NC-MIMO MC-CDMA systems, the deactivation of some of the subcarriers causes the spreading codes to be nonorthogonal and hence the challenginig issue of the joint presence of MUI and CSI is severed. Carrier interferometry (CI) codes [7] are complex orthogonal spreading codes that can be generated with any integer valued lengths. The utilization of the CI codes is investigated in [5] for the $\mathrm{NC}$ single-input single-output MC-CDMA systems.

In this paper, we model the downlink NC-MIMO MCCDMA system to be deployed in CR transmission. The two linear SVD based receivers, TIC and SC-MMSE are modified to be applicable to non-contiguous transmission when some of the subcarriers are deactivated. Also, the CI codes are deployed to solve the non-orthogonality of the conventional Hadamard-Walsh codes. So we call these new receivers as CITIC and CI-SC-MMSE. It is shown, by simulations, that the proposed NC CI-TIC and CI-SC-MMSE keep their interference mitigating performance when the number of 
deactivated subcarriers is increased. So they are very good choices for any CR based reconfigurable multi-user broadband systems.

The paper is organized as follows. After introduction, downlink MIMO MC-CDMA system model based on spacefrequency spreading code and the SVD is introduced in Section II. The CI-TIC method is developed in Section III. The SVD-based symbol-chip level MMSE method is derived in Section IV. Performance evaluations and comparisons are considered in Section $\mathrm{V}$ by computer simulations and the paper is concluded in Section VI.

\section{SYSTEM MODEL}

A downlink MIMO MC-CDMA system with $K$ users and $L$ subcarriers is considered where the base station uses $N$ transmit antennas and each of the users employ $M$ receive antennas. It is assumed that $M \geq N$ [2]. In this section, without taking into account the $\mathrm{CR}$ transmission, we briefly introduce the downlink MIMO MC-CDMA model based on the linear TIC receiver[3].

After modulating the data bits, the data vector of the $m$ th user at the base station is formed as $\mathbf{d}_{m}=\left[d_{m, 1}, d_{m, 2}, \ldots, d_{m, J}\right]^{T}$ where $J$ is the number of substreams, $J \in\{1, . ., N\}$. We assume that data symbols of different streams of different users are i.i.d with normalized power, so we have $E\left[\mathbf{d}_{m} \mathbf{d}_{k}^{H}\right]=\mathbf{I}_{J} \delta(m-k)$ for $k, m \in\{1, . ., K\}$ where $\mathbf{I}_{J}$ is the identity matrix with the size $J \times J$. In conventional MCCDMA systems, each of the data symbols is spreaded in the frequency domain (i.e., on $L$ subcarriers). However the spreading can be performed jointly on space and frequency to benefit from spatial diversity and design the ISI rejecting receivers for MIMO MC-CDMA. The space-frequency (SF) spreading code matrix is $\mathbf{C}_{m}=\left[\mathbf{c}_{m, 1}, \mathbf{c}_{m, 2}, \ldots, \mathbf{c}_{m, J}\right]$ with the size of $N L \times J$ that consists of $J$ different spreading orthogonal code vectors and we have $\mathbf{C}_{m}^{H} \mathbf{C}_{k}=\gamma \mathbf{I}_{J} \delta(m-k)$ for $m, k \in\{1,2, \ldots, K\}$ where $\gamma=N L$ is the processing gain.

The symbols of all the users are spreaded on $N L$ spacefrequency subchannels and after adding theses spreaded data, the following data vector is sent by the base station:

$\mathbf{x}_{S F D}=\sum_{k=1}^{K} \mathbf{C}_{k} \mathbf{d}_{k}$

At the receiver of the $m$ th user, the signal vector at the output of the fast Fourier transform (FFT) block becomes

$\mathbf{y}_{m}=\mathbf{H}_{m} \sum_{k=1}^{K} \mathbf{C}_{k} \mathbf{d}_{k}+\zeta_{m}$

Where $\mathbf{y}_{m}$ is the $M L \times 1$ received signal vector and $\zeta_{m}$ is the additive white Gaussian noise vector with zero mean and covariance matrix $\mathbf{R}_{\zeta}=\sigma_{\zeta}^{2} \mathbf{I}_{M L}$. Also $\mathbf{H}_{m}$ is the $M L \times N L$ block-diagonal space-frequency channel matrix between the base station and the $m$ th user. To highlight the joint presence of the CSI and MUI, $\mathbf{y}_{m}$, can be rewritten as

$\mathbf{y}_{m}=\overbrace{\mathbf{H}_{m} \mathbf{C}_{m} \mathbf{d}_{m}}^{\text {desired signal }+C S I}+\mathbf{H}_{m} \overbrace{\sum_{\substack{k=1 \\ k \neq m}}^{K} \mathbf{C}_{k} \mathbf{d}_{k}}^{M U I}+\overbrace{\zeta_{m}}^{\text {noise }}$

A powerful tool for mitigating CSI is the use of SVD based beamforming. The singular value decomposition of the block diagonal channel matrix, $\mathbf{H}_{m}$, is as follows

$\mathbf{H}_{m}=\mathbf{U}_{m} \boldsymbol{\Lambda}_{m} \mathbf{V}_{m}^{H}$

In which $\mathbf{U}_{m}$ and $\mathbf{V}_{m}$ are $M L \times N L$ and $N L \times N L$ block unitary matrices and $\boldsymbol{\Lambda}_{m}$ is the diagonal $N L \times N L$ matrix. In the SVD based receiver TIC, it is sufficient to deploy the beamforming and equalization matrices at the receiver and there is no need to deploy the matrix $\mathbf{V}_{m}$ as the transmit beamforming matrix for the $m$ th user. So we have

$\tilde{\mathbf{y}}_{m}=\boldsymbol{\Lambda}_{m}^{-1} \mathbf{U}_{m}^{H} \mathbf{y}_{m}=\mathbf{V}_{m}^{H} \sum_{k=1}^{K} \mathbf{C}_{k} \mathbf{d}_{k}+\boldsymbol{\Lambda}_{m}^{-1} \mathbf{U}_{m}^{H} \zeta_{m}$

To finalize the joint CSI-MUI rejection, we deploy the despreading matrix at the receiver that satisfies the following relation

$\mathbf{G}_{m}^{H}=\mathbf{C}_{m}^{H} \mathbf{V}_{m}$

where the size of $\mathbf{G}_{m}$ is $N L \times J$. After despreading, we have

$\hat{\mathbf{d}}_{m}=\frac{1}{N L} \mathbf{G}_{m}^{H} \tilde{\mathbf{y}}_{m}=\mathbf{d}_{m}+\boldsymbol{\eta}_{m}$

where $\boldsymbol{\eta}_{m}=\mathbf{G}_{m}^{H} \boldsymbol{\Lambda}_{m}^{-1} \mathbf{U}_{m}^{H} \boldsymbol{\zeta}_{m}$ is the $J \times 1$ noise vector at the output of the despreader. As it can be seen in (7), both CSI and MUI are completely mitigated and total interference becomes zero; consequently, the proposed method is called total interference cancellation (TIC).

\section{CI-TIC RECEIVER FOR NC-MIMO MC-CDMA}

In cognitive radio systems, the secondary users should use the part of the spectrum that is not used by the primary user. Consider the case where $P$ subcarriers out of total $L$ subcarriers are occupied by the primary user. For the secondary MC-CDMA users, these subcarriers are turned off to avoid the interference and the base station should spread the users' data symbols on only $I=L-P$ subcarriers. In NC-MIMO MC-CDMA, the data symbols of each user are spreaded on $N I$ space-frequency subchannels that could be any integer value and therefore allowing maximum $K_{\text {full-load }}=I$ distinct users. The orthogonality of the conventional spreading codes is applicable only when their length has a specific value. The well-known Hadamard-Walsh codes only exist when $I$ is a multiple of 4 . To solve this challenging issue, we deploy the complex carrier interferometry code set that its orthogonality property is independent of the codes length.

Let the CI spreading code matrix of the $k$ th user be 
$\mathbf{C}^{\prime}{ }_{k}=\left[\mathbf{c}_{k, 1}^{\prime}, \mathbf{c}_{k, 2}^{\prime}, \ldots, \mathbf{c}_{k, J}^{\prime}\right]$ with the size of $N I \times J$. The $j$ th column of $\mathbf{C}_{m}^{\prime}$ is defined as

$\mathbf{c}_{k, j}^{\prime}=\left[e^{j 0}, e^{j \theta_{k, j}}, \ldots, e^{j(N I-1) \theta_{k, j}}\right]^{T}$

where the phase of the code vector is set as follows

$\theta_{k, j}=\frac{2 \pi}{N I} k^{\prime}, \quad 0 \leq k^{\prime} \leq N I-1$

where

$k^{\prime}=(k-1) J+j, \quad 1 \leq k \leq I, 1 \leq j \leq J$

is the index of the substream. It can be seen that the code length is $N I$. The CI codes are orthogonal, so we have

$\mathbf{C}^{\prime H}{ }_{m}^{\prime} \mathbf{C}_{k}^{\prime}=\gamma \mathbf{I}_{J} \delta(m-k)$

for $m, k \in\{1,2, \ldots, K\}$ and $\gamma=N I$ is the new processing gain .

By spreading the data vector of all users, the SF data vector of the active subcarriers becomes

$\mathbf{x}_{S F D}^{\prime}=\sum_{k=1}^{K} \mathbf{C}_{k}^{\prime} \mathbf{d}_{k}=\left[\mathbf{x}_{S F D}^{\prime(1) T}, \mathbf{x}_{S F D}^{\prime(2) T}, \ldots, \mathbf{x}_{S F D}^{\prime(I) T}\right]^{T}$

where the size of $\mathbf{x}_{S F D}^{\prime}$ is $N I \times 1$ and $\mathbf{x}_{S F D}^{\prime(i)}$ represents the spatial $N \times 1$ data vector on the $i$ th active subcarrier.

To deploy $L$-point IDFT at the transmitter, The $N I \times 1$ data vector, $\mathbf{x}_{S F D}^{\prime}$, is mapped to active subcarriers of the $N L \times 1$ data vector, $\mathbf{x}_{S F D}=\operatorname{Map}\left(\mathbf{x}_{S F D}^{\prime}\right)$. Let the subset determining the indexes of the available subcarriers to be $F=\left\{f_{1}, f_{2}, \ldots f_{I}\right\} \quad$ where $f_{i} \in\{1, \ldots, L\}$. By deploying MATLAB $^{\mathrm{TM}}$ syntax for referring to the rows and column of the matrices, we have

$\left\{\begin{array}{c}\mathbf{x}_{S F D}\left(n f_{i}, 1\right)=\mathbf{x}_{S F D}^{\prime}(n i, 1), \quad f_{i} \in F \\ \mathbf{x}_{S F D}(n g, 1)=0 \quad \text { if } g \notin F\end{array} \quad\right.$ for $n \in\{1, \ldots, N\}$

The data vector $\mathbf{x}_{S F D}$ can also be written as

$\mathbf{x}_{S F D}=\left[\mathbf{x}_{S F D}^{(1) T}, \mathbf{x}_{S F D}^{(2) T}, \ldots, \mathbf{x}_{S F D}^{(L) T}\right]^{T}$

in which $\mathbf{x}_{S F D}^{(l)}$ represents the spatial $N \times 1$ data vector on the $l$ th subcarrier. To transmit $\mathbf{x}_{S F D}$, the subcarriers of each antenna branch is selected by the $N L \times N L$ selection matrix $\mathbf{P}_{N L}$ where for example $\mathbf{P}_{2 L}$ is defined as

$\left.\mathbf{P}_{2 L}=\left[\begin{array}{ccccccc}\overbrace{1}^{l=1} & 0 & 0 & 0 & \cdots & 0 & 0 \\ 0 & 0 & 1 & 0 & \cdots & 0 & 0 \\ \vdots & & \vdots & \ddots & \vdots & \vdots=2 \\ 0 & 0 & 0 & 0 & \cdots & 1 & 0 \\ 0 & 1 & 0 & 0 & \cdots & 0 & 0 \\ 0 & 0 & 0 & 1 & \cdots & 0 & 0 \\ \vdots & & \vdots & \ddots & \vdots \\ 0 & 0 & 0 & 0 & \cdots & 0 & 1\end{array}\right]\right\} n=1$

So the time-domain (TD) data vector can be written as
$\mathbf{x}_{T D}=\frac{\sqrt{L}}{\sqrt{I}} \mathbf{W}_{B D}{ }^{H} \mathbf{P}_{N L} \mathbf{x}_{S F D}=\left[\mathbf{x}_{T D}^{[1] T}, \mathbf{x}_{T D}^{[2] T}, \ldots, \mathbf{x}_{T D}^{[N] T}\right]^{T}$

where $\mathbf{W}_{B D}$ is the $N L \times N L$ block diagonal DFT matrix as follows

$\mathbf{W}_{B D}=\operatorname{block} \operatorname{diag}\left(\mathbf{W}_{L \times L}, \ldots, \mathbf{W}_{L \times L}\right)$

and $\mathbf{W}_{L \times L}$ is the $L \times L$ FFT matrix that its $(i, j)$ th element is $\left[\mathbf{W}_{L \times L}\right]_{i, j}=\frac{1}{\sqrt{L}} e^{-2 \pi \sqrt{-1} \frac{(i-1)(j-1)}{L}}$. In (16), the coefficient $\frac{\sqrt{L}}{\sqrt{I}}$ is used to allocate the available power to the active subcarriers. After adding cyclic prefix (CP) and upconversion, $\mathbf{x}_{T D}$, is transmitted by $N$ antennas.

At the receiver, after removing $\mathrm{CP}$ and DFT and discarding the turned-off subcarriers, the $N I \times 1$ space-frequency data vector for the active subchannels becomes

$\mathbf{y}_{m}^{\prime}=\mathbf{H}_{m}^{\prime} \sum_{k=1}^{K} \mathbf{C}_{k}^{\prime} \mathbf{d}_{k}+\zeta_{{ }_{m}}^{\prime}$

where $\mathbf{H}_{m}^{\prime}$ is the $M I \times N I$ space-frequency block diagonal channel matrix that is attained by discarding the subchannel matrices of the turned off subcarriers in $\mathbf{H}_{m}$. Similar to (4), the SVD of $\mathbf{H}^{\prime}{ }_{m}$ can be obtained as follows

$\mathbf{H}_{m}^{\prime}=\mathbf{U}_{m}^{\prime} \boldsymbol{\Lambda}_{m}^{\prime} \mathbf{V}_{m}^{\prime H}$

In which $\mathbf{U}_{m}^{\prime}$ and $\mathbf{V}_{m}^{\prime}$ are $M I \times N I$ and $N I \times N I$ block unitary matrices and $\Lambda_{m}^{\prime}$ is the diagonal $N I \times N I$ matrix. Deploying the beamformer, equalizer and despreading matrices respectively, we have

$\hat{\mathbf{d}}_{m}=\frac{1}{N L} \mathbf{G}_{m}^{\prime H} \boldsymbol{\Lambda}_{m}^{\prime-1} \mathbf{U}_{m}^{\prime H} \mathbf{y}_{m}^{\prime}=\mathbf{d}_{m}+\frac{1}{N L} \mathbf{G}_{m}^{\prime H} \boldsymbol{\Lambda}_{m}^{\prime-1} \mathbf{U}_{m}^{\prime H} \zeta_{m}^{\prime}$

The despreading matrix, $\mathbf{G}_{m}^{\prime}$, is calculated as

$\mathbf{G}_{m}^{\prime H}=\mathbf{C}_{m}^{\prime H} \mathbf{V}_{m}^{\prime}$

Note that in (20), the despreading coefficient is $\frac{1}{N L}$, because the available power is only allocated to the active subcarriers, as can be seen in (16).

Although some of the subcarriers are deactivated, by deploying the orthogonal complex CI codes that exist for any integer-valued length, the ability of CI-TIC to completely eliminating CSI and MUI is retained. As it can be seen in (20), the SF noise at the input of the slicer is multiplied with the inverse of related singular values of the channel matrix at that subchannel. So small singular values degrade the performance of the CI-TIC method. In the next section, we develop another method based on MMSE criterion to address this challenging issue that can also be applicable in CR scenario.

\section{CI SYMBOL-CHIP LEVEL MMSE METHOD}

Consider the received SF data vector in (18), in this method, 
firstly the beamforming matrix, $\mathbf{U}_{m}^{\prime H}$, is utilized as follows

$\tilde{\mathbf{y}}_{m}^{\prime}=\mathbf{U}_{m}^{\prime H} \mathbf{y}_{m}^{\prime}$

To avoid the noise amplification due to small singular values of $\mathbf{\Lambda}_{m}^{\prime-1}$, the $N I \times N I$ diagonal equalization matrices are separately deployed for each substream. So the $j$ th received datastream after despreading becomes

$\hat{d}_{m, j}=\mathbf{g}_{m, j}^{\mathbf{\prime}^{H}} \mathbf{F}_{m, j}^{H} \tilde{\mathbf{y}}_{m}^{\prime}$

In order to calculate the equalizer coefficients, we rewrite (23) by swapping the depsreader and the equalizer as

$$
\begin{aligned}
\hat{d}_{m, j} & =\mathbf{f}_{m, j}^{H} \tilde{\mathbf{G}}_{m, j}^{\prime H} \tilde{\mathbf{y}}_{m}^{\prime}=\mathbf{f}_{m, j}^{H} \tilde{\mathbf{G}}_{m, j}^{\prime H} \boldsymbol{\Lambda}_{m}^{\prime} \mathbf{V}_{m}^{\prime H} \mathbf{C}_{m}^{\prime} \mathbf{d}_{m} \\
& +\mathbf{f}_{m, j}^{H} \tilde{\mathbf{G}}_{m, j}^{\prime H} \boldsymbol{\Lambda}_{m}^{\prime} \mathbf{V}_{m}^{\prime H} \sum_{\substack{k=1 \\
k \neq m}}^{K} \mathbf{C}_{k}^{\prime} \mathbf{d}_{k}+\mathbf{f}_{m, j}^{H} \tilde{\mathbf{G}}_{m, j}^{\prime H} \mathbf{U}_{m}^{\prime H} \zeta_{m}^{\prime}
\end{aligned}
$$

In the above relation, the first term represents the desired symbol of the $m$ th user, the second and the third terms are CSI and MUI respectively and the last term represents the noise part. To compute the equalization vector, $\mathbf{f}_{m, j}$ that is utilized at the chip level, the MMSE criterion is employed after despreading process at the symbol level. So, we call this method as symbol-chip level MMSE. The mean square error at symbol-level is defined as follows

$$
\varepsilon=E\left[\left\|d_{m, j}-\hat{d}_{m, j}\right\|^{2}\right]
$$

By minimizing $\varepsilon$ with respect to $\mathbf{f}_{m, j}$ and after doing some manipulations, we have

$$
\mathbf{f}_{m, j}=\left(\mathbf{A}_{m, j}+\mathbf{B}_{m, j}\right)^{-1} \mathbf{a}_{m, j}
$$

where $\mathbf{A}_{m, j}, \mathbf{B}_{m, j}$ and $\mathbf{a}_{m, j}$ are defined as

$$
\begin{aligned}
\mathbf{A}_{m, j} & =\tilde{\mathbf{G}}_{m, j}^{\prime H} \boldsymbol{\Lambda}_{m}^{\prime} \mathbf{V}_{m}^{\prime H}\left(\sum_{k=1}^{K} \mathbf{C}_{k}^{\prime} \mathbf{C}_{k}^{\prime H}\right) \mathbf{V}_{m}^{\prime} \boldsymbol{\Lambda}_{m}^{\prime} \tilde{\mathbf{G}}_{m, j}^{\prime} \\
\mathbf{B}_{m, j} & =\sigma_{\zeta}^{2} \tilde{\mathbf{G}}_{m, j}^{\prime H} \tilde{\mathbf{G}}_{m, j}^{\prime} \\
\mathbf{a}_{m, j} & =\tilde{\mathbf{G}}_{m, j}^{\prime H} \boldsymbol{\Lambda}_{m}^{\prime} \mathbf{V}_{m}^{\prime H} \mathbf{c}_{m, j}^{\prime}
\end{aligned}
$$

The NC-CI-SC-MMSE receiver eliminates the deteriorating effect of small singular values of the channel matrix. Therefore, comparing equations (24) for SC-MMSE with (20) for the TIC, it can be seen that the orthogonality property is not preserved and thus the MUI is not completely eliminated in the symbol-chip MMSE method. However, the effect of interference plus noise on detection at the symbol level is minimized, resulting in superior performance.

\section{SIMULATIONS AND COMPARISONS}

A MIMO MC-CDMA system for downlink communication is considered in simulations when $J=M=N=2$ and the number of subcarriers is $L=32$. A sequence of independent, identically distributed signal vector with 16QAM modulation scheme is sent from transmitter antenna arrays. Each frequency selective MIMO channel between the base station and each user has been realized based on an exponential power delay profile with $L_{\mathrm{c}}=8$ resolvable paths where the exponential decay factor is assumed to be $\beta=0.1$.

The proposed NC-CI receivers, namely CI-TIC and CI-SCMMSE, are compared with the case where the HadamardWalsh (HW) codes are deployed instead of CI codes that we call them as HW-TIC and HW-SC-MMSE. Since theses codes don't exist when the code length is not a multiple of four, the Hadamard Walsh codes are generated whose lengths are the smallest multiple of four that is larger than the required length and then they are cut off to the desired length. The performance evaluation is done based on attained BER in different $\mathrm{CR}$ scenarios and single-user performance is also included as the benchmark.

In Fig.1, the bit error rate performances of the four methods are compared when $P=8$ subcarriers are utilized by the primary users. So the number of active subcarriers is $I=L$ $P=24$ and hence in the full-loaded case we can have $K=I=24$ active users. It can be seen that the TIC and SC-MMSE receivers can be utilized in the non-contiguous MIMO MCCDMA where some of the subcarriers are deactivated. The TIC receivers completely eliminate multiuser interference however suffer performance degradation due to small singular values of the channel. The SC-MMSE receiver solves this drawback and shows superior BER performance both in the single user and full-loaded scenarios, since the SC-MMSE doesn't deploy $\boldsymbol{\Lambda}_{m}^{-1}$ as the equalization matrix. In this scenario, as the codes length, $N I=48$, is multiple of four, the HW codes are orthogonal with no performance loss. The CI receivers show the same performance of the HW receivers proving the applicability of the complex CI codes instead of the orthogonal antipodal HW codes for downlink MIMO MCCDMA transmission.

In Fig. 2 we have considered the case where $P=9$ subcarriers are turned-off. Since $N I=46$ is not multiple of four, the HW codes aren't orthogonal and significant error floor appears at $\mathrm{BER}=10^{-2}$ for HW-TIC. On the other hand, the CI-TIC ability to MUI rejection is maintained because of using the CI codes. For the SC-MMSE receivers, it can be seen that in the single user case, the performance of both the receivers is the same but in the fully loaded environment, SC-MMSE with $\mathrm{HW}$ codes shows $5 \mathrm{~dB}$ performance loss due to MAI arising to the system at $\mathrm{BER}=10^{-2}$ with respect to CI-SC-MMSE receiver. However HW-SC-MMSE doesn't experience any error floor comparing to HW-TIC. It shows that the SCMMSE is much stronger than the TIC receiver to cope with the interferences even when the codes are not orthogonal.

The BER performance of the proposed receivers is depicted in Fig.3, when the number of deactivated subcarriers is increased to $P=21$, so only $I=11$ subcarriers are available. In this case the codes length $N I=22$ is not multiple of 4 . Comparing Figs. 2 and 3, it can be seen that the performance of the CI-TIC receiver is slightly degraded in high SNRs only. Because with 
less available subcarriers, the diversity order is decreased. However, roughly speaking, the CI-TIC performance doesn't depend on the number of active subcarriers. But the BER performance of both SC-MMSE receivers depends on the number of active subcarriers. The performance of CI-SCMMSE and HW-SC-MMSE receivers is degraded about $5 \mathrm{~dB}$ at $\mathrm{BER}=10^{-4}$ in the full-loaded scenario.

\section{CONCLUSIONS}

The Multiuser interference, co-space interference and the loss of spreading codes orthogonality due to deactivation of some of the subcarriers are the challenging issues ahead of MIMO MC-CDMA systems for being utilized by the secondary users in downlink CR transmission. In this paper, two SVD based linear receivers are developed for CR transmission that deploy carrier interferometry spreading codes to cope with the deactivation of the subcarriers. The first method, CI-TIC is able to mitigate the MUI and the CSI almost independently of number of available subcarriers. It is shown that when the TIC is developed by using Hadamard Walsh codes, there will occur error floor in its BER performance in the fully loaded case.

Although the CI-TIC completely eliminates CSI and MUI, it amplifies the noise power. So the CI-SC-MMSE is also developed by considering both the interference and noise as well as the deactivation of the subcarriers. The CI-SC-MMSE method shows better performance in comparison with the CITIC due to using MMSE criterion to design equalizer weights. However, in contrast to CI-TIC, its performance is degraded when the number of deactivated subarriers is increased.

\section{REFERENCES}

[1] T. Weiss and F. Jondral, "Spectrum pooling: an innovative strategy for the enhancement of spectrum efficiency," IEEE Commun. Mag., vol. 42, pp. 8-14, March 2004.

[2] M. Vehkapera, D. Tujkovic, Z. Li and M. Juntti, "Receiver design for spatially layered downlink MC-CDMA system, "IEEE Trans. Vehic. Technol., vol. 54, No. 3, pp. 1042 - 1055, May 2005.

[3] H. Zamiri-Jafarian, M. Rajabzadeh, "SVD-based receiver for downlink MIMO MC-CDMA systems," IEEE ICC, June 2009, pp. 1-5.

[4] A. Phasouliotis and D.K.C. So, "A novel OSSMIC receiver for downlink MIMO MC-CDMA systems," ," in Proc. IEEE VTC, May 2008, pp. 1271-1275.

[5] Z. Wu, P. Ratazzi, V. D. Chakravarthy and Lang Hong, "Performance evaluation of adaptive non-contiguous MC-CDMA and non-contiguous CI/MC-CDMA for dynamic spectrum access", IEEE CrownCom, May 2008, pp.1-6

[6] R. Rajbanshi, et al., "Quantitative comparison of agile modulation techniques for cognitive radio transceivers," IEEE CCNC, Jan. 2007, pp. 1144-1148.

[7] B. Natarajan, C. R. Nassar, M. Michelini, and Z. Wu, "Highperformance MC-CDMA via carrier interferometry codes," IEEE Trans. Veh. Technol., vol. 50, no. 6, pp. 1344-1353, Nov. 2001.

\section{ACKNOWLEDGMENT}

This work was supported in part by Iran Telecom Research Center (ITRC).

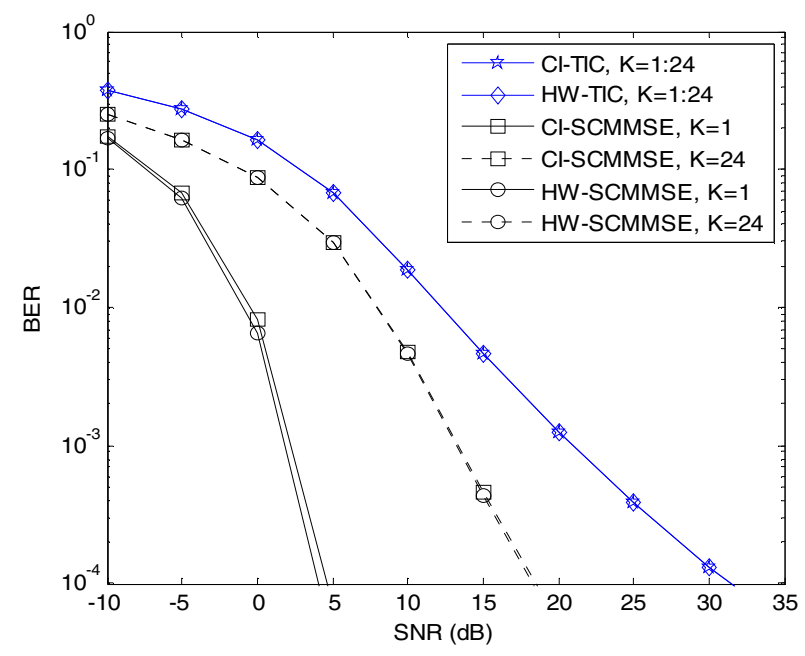

Fig.1. BER versus SNR for the TIC and the SCMMSE methods when $I=24$.

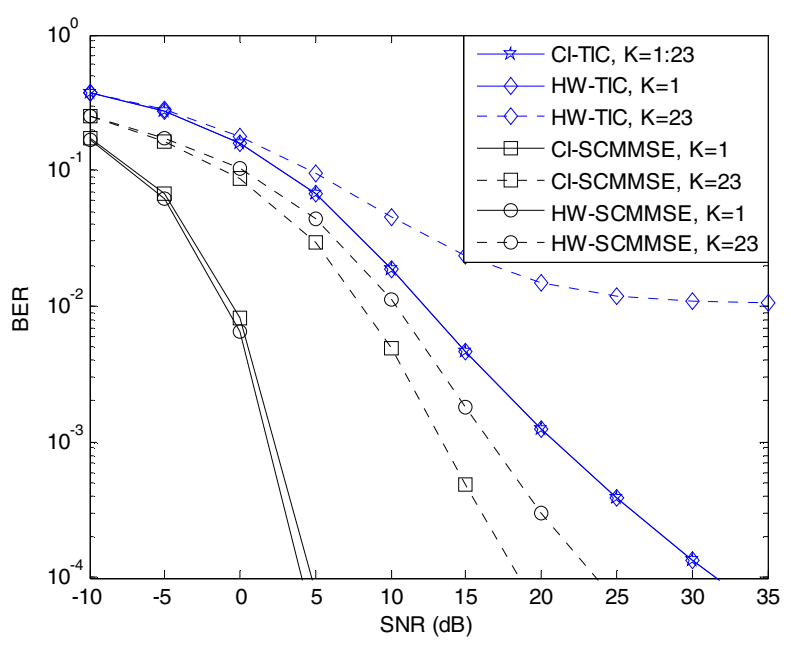

Fig.2. BER versus SNR for the TIC and the SCMMSE methods when $I=23$.

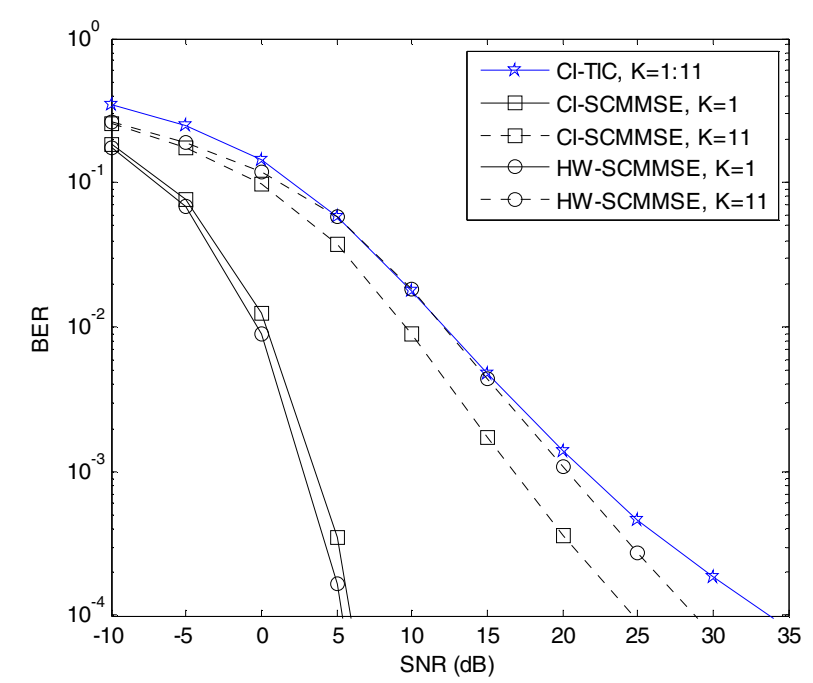

Fig.3. BER versus SNR for the CI-TIC and the SCMMSE methods when $I=11$. 